\title{
Evaluation of an interferon-gamma release assay in young contacts of active tuberculosis cases
}

\author{
S. Noorbakhsh, ${ }^{1}$ J. Mousavi, ${ }^{7}$ M. Barati, ${ }^{1}$ A. R. Shamshiri, ${ }^{2}$ M. Shekarabi, ${ }^{1}$ A. Tabatabaei ${ }^{1}$ and Gh. Soleimani ${ }^{3}$
}

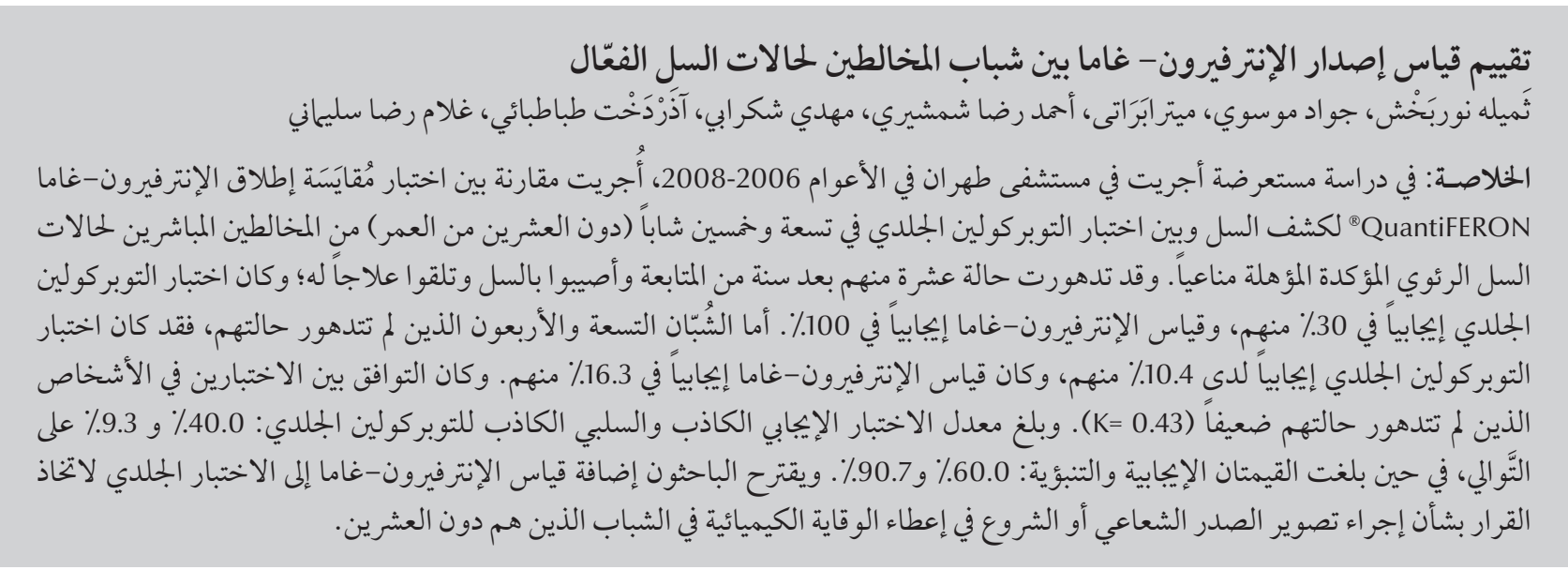

ABSTRACT In a cross-sectional study in a hospital in Tehran in 2006-08 the QuantiFERON ${ }^{\circledR}$-TB interferon-gamma release assay (QTB) was compared with the tuberculin skin test (TST) in 59 young people (aged $<20$ years) with close contact with immunocompetent cases of proven pulmonary tuberculosis. After 1 year follow-up 10 subjects had progressed to tuberculosis disease and received treatment; TST was positive in 30\% and QTB in 100\%. Of the 49 non-progressive subjects, TST was positive in 10.4\% and QTB in 16.3\%. The agreement between TST and QTB assay in non-progressive subjects was poor $(\mathcal{K}=0.43)$. False positive and false negative rates for TST were $40.0 \%$ and $9.3 \%$ respectively; positive and predictive values were $60.0 \%$ and $90.7 \%$. We suggest adding the interferon assay to the skin test in the decision to perform chest X-ray or to start chemoprophylaxis at least in younger subjects (aged $<20$ years).

Évaluation portant sur la mesure de la production d'interféron gamma chez de jeunes contacts de patients atteints de tuberculose active

RÉSUMÉ Dans le cadre d'une étude transversale conduite dans un hôpital de Téhéran de 2006 à 2008, le test QuantiFERON ${ }^{\circledR}$-TB de mesure de la production d’interféron gamma a été comparé au test tuberculinique cutané chez 59 jeunes âgés de moins de 20 ans en contact rapproché avec des cas immunocompétents de tuberculose pulmonaire confirmés. Après un an de suivi, dix contacts avaient évolué vers une tuberculose et recevaient un traitement ; $30 \%$ d'entre eux avaient un résultat positif au test tuberculinique cutané, et $100 \%$ au test QuantiFERON ${ }^{\circledR}$-TB. Sur les 49 sujets sans évolution, 10,4 \% avaient un résultat positif au test tuberculinique cutané, et 16,3\% au test QuantiFERON ${ }^{\circledR}$-TB. La concordance entre les deux tests chez les sujets non évolutifs était médiocre $(\mathcal{K}=0,43)$. Les taux de faux positifs et de faux négatifs pour le test tuberculinique cutané s'élevaient respectivement à 40,0\% et 9,3\% ; les valeurs positives et prédictives étaient de 60,0\% et 90,7\%. Nous suggérons d'ajouter la mesure de la production d'interféron gamma au test tuberculinique cutané pour prendre ou non la décision de réaliser une radiographie des poumons ou d'entamer une chimioprophylaxie, au moins chez les sujets jeunes (âgés de moins de 20 ans).

${ }^{\prime}$ Research Centre of Paediatric Infectious Diseases, Tehran University of Medical Sciences, Tehran, Islamic Republic of Iran (Correspondence to S. Noorbakhsh:samileh_noorbakhsh@yahoo.com).

${ }^{2}$ Department of Epidemiology and Biostatistics, School of Public Health, Tehran University of Medical Sciences, Tehran, Islamic Republic of Iran. ${ }^{3}$ Department of Paediatric Infectious Diseases, Zahedan University of Medical Sciences, Zahedan, Islamic Republic of Iran.

Received: 22/11/09; accepted: 21/02/10 


\section{Introduction}

Tuberculosis (TB) is still one of the major causes of mortality and morbidity $[1,2]$. Treatment of latent TB infection is very important, especially in recently infected children, to prevent progression to active disease. The tuberculin skin test (TST) is a screening test universally used for diagnosis of latent $\mathrm{TB}$ in many countries. The advantages and disadvantages of TST are well known and include the booster phenomenon, technical difficulties, the need for repeat visits and a high number of false positive and false negative results. False negatives are frequent in immunocompromised cases. However, in young children false negative results cannot be detected. False positive TST occurs in individuals who have been vaccinated with BCG or infected with non-TB Mycobacterium spp. $[3,4]$.

The Islamic Republic of Iran is an intermediate TB burden country. Bacille Calmette-Guérin (BCG) vaccination is given at birth and at school entrance (6 years old). Due to a number of reasons the second BCG vaccination was stopped 3 years ago [5]. Finding a better indicator than the TST for detecting the risk of $M$. tuberculosis infection in our country is needed. Such a test would lead to a reduction in the cost and unnecessary chemoprophylaxis of children in close contact with TB cases [6-8].

QuantiFERON ${ }^{\circ}$-TB (QTB) is an in vitro whole-blood interferon-gamma release assay, which is a novel alternative to the TST and is now recommended as the gold standard test for diagnosis of TB infection [9]. It has been used in decision-making about chemoprophylaxis treatment for those in close contact with $\mathrm{TB}$ cases or the diagnosis of latent $\mathrm{TB}$ infection $[6-8,10-15]$. There are little data, however, about its use in children.

The following study was performed to detect the agreement between TST and QTB in young household contacts (aged $<20$ years) of cases of proven active pulmonary TB in a BCG-vaccinated population in Tehran, Islamic Republic of Iran, and to compare subjects progressing to $\mathrm{TB}$ with non-progressive subjects.

\section{Methods}

\section{Study setting and sample}

This cross-sectional study was done in the pulmonary and infectious diseases department of Rasul hospital in Tehran, 2006-08. Our centre is a tertiary care general hospital with 500 active beds and 2 research centres for pulmonary and infectious diseases. This study was approved by the ethical committee of the Iran University of Medical Sciences.

The study subjects were all young ( $<20$ years old) household contacts of people with confirmed active pulmonary $\mathrm{TB}$ and previous BCG vaccination received at birth. The subjects were invited to our research centre for clinical and laboratory follow-up. The index cases were all documented active pulmonary TB cases (aged $>15$ years) who had been diagnosed by an internist in the pulmonary and infectious ward of Rasht hospital. The index cases were confirmed by positive culture for $M$. tuberculosis or sputum smear-positive TB. Close or household contact was defined as any person who had lived with the index case for more than 3 months. History of BCG vaccination was confirmed by BCG scar or vaccination chart examined by physicians. Household contacts were excluded if they had been treated for $\mathrm{TB}$ in the past year or had a known immunodeficiency state on history or clinical signs (malignancy, corticosteroid therapy, HIV, etc.).

Over the study period we located 59 immunocompetent young people who were household contacts of 55 patients with proven active pulmonary $\mathrm{TB}$.

\section{Data collection}

A questionnaire was completed by an authorized physician for each study subject followed by a complete clinical examination. After collection of blood samples for the QTB assay, all participants underwent TST.

For the TST a test dose $(0.1 \mathrm{~mL})$ of 5 tuberculin units of purified protein derivative solution (Pasteur Institute, Tehran) was injected intradermally into the volar aspect of the forearm with a 26-27 gauge needle by trained field worker. The induration diameter of the raised, blanched weal (not the erythema) was read after 48-72 hours. A reactive TST was an induration diameter of $\geq 10 \mathrm{~mm}[1-4,9]$.

For the QTB fresh blood samples from all of the participants were processed on site according to the manufacturer's instruction (Gold Quantiferon-TB, Cellestis). First, 1 $\mathrm{mL}$ of heparinized whole blood was incubated with aliquots of antigen-free control and antigens for 16-24 hours at $37^{\circ} \mathrm{C}$ in a carbon dioxide incubator. After overnight incubation, $200 \mu \mathrm{L}$ plasma was removed from each well and the concentration of IFN- $\gamma$ was determined using the assay kits.

Subjects with a reactive TST or QTB assay were offered a chest radiograph and those with symptoms were assessed using a standardized proform and clinical examination $[1-4,9]$. Those with TB disease were referred to the national TB control programme for free treatment following World Health Organization recommendations $[1,2]$. All the subjects were followed for at least 1 year.

\section{Statistical analysis}

Quantitative variablesweresummarized as mean and standard deviation (SD) and qualitative variables as counts with percentage. Comparison of the age distribution between subjects with positive or negative test results was performed by Mann-Whitney test because the number of positive subjects was small. Comparison of the sex distribution was assessed by chi-squared test (or Fisher exact test if necessary). 
The percentage concordance between TST and QTB was reported as actual percentage agreements and the degree of agreement between the 2 tests was reported as Cohen kappa $(\kappa)$ statistic in the following categories: excellent $(\kappa>0.75)$, fair to good $(\kappa=0.4-0.75)$ or poor agreement $(\kappa<0.4)$.

All analyses were conducted using SPSS, version 11.5 and $P$-values $<0.05$ were considered statistically significant.

\section{Results}

During the study period, 10 of the 59 study subjects progressed to $\mathrm{TB}$ disease and received anti-TB drugs. The mean age of the progressed subjects was 5.4 (SD 3.3) years. The female to male ratio was $7 / 3$. All of the progressed cases (10/10) had positive QTB results, but only $30.0 \%(3 / 10)$ had reactive TST (Table 1). The mean age of the remaining 49 non-progressive subjects was 8.0 (SD 6.7) years (Figure 1), with a female to male ratio of $27 / 21$; we had sex for 1 case missing. A positive QTB was reported in $16.3 \%(8 / 49)$ and reactive TST in $10.4 \%(5 / 48)$ of subjects. The case with sex missing was negative for both TST and QTB.

Although positive TST subjects were older than those with negative TST the difference was not statistically significant [mean age 13.4 (SD 6.7) years versus $7.5(\mathrm{SD} 6.6)$ years $](P=$
0.53). The mean age of subjects with positive and negative QTB was also not significantly different $[11.3$ (SD 7.3) years versus $7.4(\mathrm{SD} 6.5)$ years $](P=$ 0.66 ).

No statistically significant difference was observed in the sex distribution between positive and negative results for both TST and QTB tests $(P=0.65$ and 0.72 respectively) (Table 1 ).

The agreement between TST and QTB assay in non-progressive subjects was poor $(\kappa=0.43, P=0.002)$; however, the actual agreement was $87.6 \%$. The rate of false positives and false negatives for TST in comparison with QTB (as the reference test) were $40.0 \%(2 / 5)$ and $9.3 \%(4 / 43)$ respectively. The positive predictive value was $60.0 \%$ (positive TST with positive QTB) and the negative predictive value was $90.7 \%$ (negative TST with negative QTB).

\section{Discussion}

The results of this study found a poor agreement between the results of the skin test and interferon assay in our young, high-risk subjects. The discrepancy was greatest in those subjects who progressed to TB disease (100\% positive QTB versus 30\% reactive TST), but it was also observed in subjects who did not progress (16.3\% positive QTB versus $10.4 \%$ reactive TST).
The false positive rate of the TST in non-progressive subjects (followed for 1 year) was $40.0 \%$. Those reactive TSTs might due to remote or non-Mycobacterium infections, which can give a TST false positive result with indurations of 5-14 mm [3]. Although prior BCG vaccination could be the reason for a false positive TST, a lower sensitivity of QTB in detecting TB infection is also plausible. The $\kappa$-value for the agreement between TST and QTB assay in non-progressive subjects was only 0.43 . Therefore as many as $57 \%$ of true recent infected cases will be missed if we use the skin test as the only TB infection screening test (not only in progressive but also in non-progressive subjects).

Our study showed that the QTB assay is an acceptable diagnostic test for determining recent $M$. tuberculosis infection in a vaccinated population. Similar results were found in at least 4 other studies $[8,9,11,15]$. An exception is an Indian study, which reported 100\% agreement $(\kappa=1.0)$ between TST and QTB in BCG scar-negative children compared with $94 \%(\kappa=0.63)$ in scarpositive children. BCG vaccination did not significantly affect either TST or QTB results [7]. There are a number of possible reasons for the difference, including the higher rate of $\mathrm{TB}$ infection in India, the lower rate of HIV infection in the Islamic Republic of Iran population and the possibly better economic

\begin{tabular}{|c|c|c|c|c|c|c|}
\hline \multirow{2}{*}{ TB disease status/test } & \multicolumn{3}{|c|}{ +ve test } & \multicolumn{3}{|c|}{-ve test } \\
\hline & No. & $\begin{array}{c}\text { Mean (SD) } \\
\text { age }\end{array}$ & $\begin{array}{c}\text { Sex ratio } \\
(\mathrm{F} / \mathrm{M})\end{array}$ & No. & $\begin{array}{c}\text { Mean (SD) } \\
\text { age }\end{array}$ & $\begin{array}{c}\text { Sex ratio } \\
(\mathrm{F} / \mathrm{M})\end{array}$ \\
\hline \multicolumn{7}{|l|}{ Progressive $(n=10)$} \\
\hline TST & 3 & $4.8(3.1)$ & $2 / 1$ & 7 & $3.5(2.8)$ & $5 / 2$ \\
\hline QTB & 10 & $5.4(3.3)$ & $7 / 3$ & 0 & - & - \\
\hline \multicolumn{7}{|l|}{ Non-progressive $(n=49)$} \\
\hline TST & 5 & $13.4(6.7)$ & $2 / 3$ & 43 & $7.5(6.6)$ & $25 / 17$ \\
\hline QTB & 8 & $11.3(7.3)$ & $5 / 3$ & 41 & $7.4(6.5)$ & $22 / 18$ \\
\hline
\end{tabular}

Sex was missing for 1 case (1/49) and TST was missing for another (1/49).

$N=$ total number of cases; $n=$ number of cases; $S D=$ standard deviation; $F=$ female; $M=$ male. 


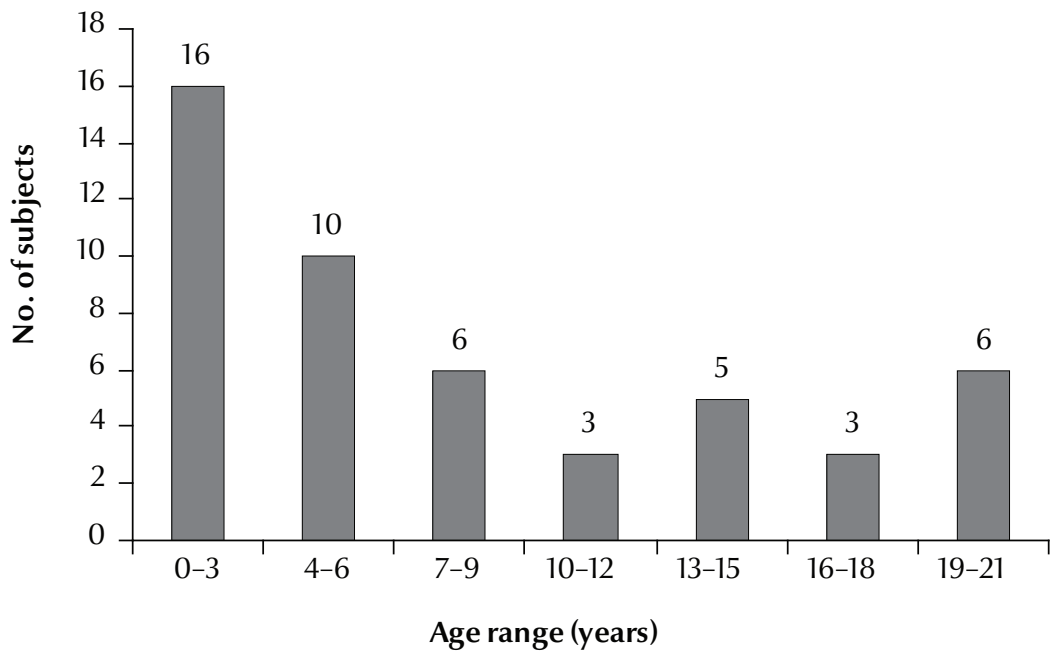

Figure 1 Age distribution of the 49 study subjects who did not progress to tuberculosis disease

and nutritional status of the subjects in the present study.

A weakness of previous studies is that, due to difficulties in obtaining accurate immunization histories and/or records, they may have relied on the presence or absence of a BCG scar to determine BCG vaccination status. In our study all the studied subjects were young, proven immunocompetent individuals. Mazurek et al. reported higher discordant results (26-40 times) between the 2 tests in recruits who were born in countries with a high prevalence of TB infection than in countries with a low prevalence [11]. A German study found a poor agreement between the TST and the QTB $(\kappa=0.2)$ and positive reactions were associated with prior $B C G$ vaccination $(O R=24.7)$. A good agreement between the 2 tests, however, has been observed in unvaccinated individuals $(\kappa=0.58)[15]$ and in countries with low rates of TB infection unvaccinated populations $[4,13,14]$.

The results of the last published study in the Islamic Republic of Iran on 20 high-risk BCG-vaccinated adults (mean age 35 years) was similar to the present study. The agreement between the 2 tests $(\kappa=0.28)$ was even lower than in our study $(\kappa=0.43)$, but a higher rate of positive QTB results was observed in older subjects $(\mathrm{OR}=1.09, P<0.001)$ [8]. In our study the mean age of subjects with positive TST was higher (13.4 years) than those with positive QTB (11.5 years), which may be due to differences in the age distribution of subjects in these 2 Iranian studies. We observed positive QTB in $16.3 \%$ of subjects, reactive TST in $10.4 \%$ of the subjects and a $6 \%$ false negative rate for the TST. Basing clinical decision-making on the TST alone would increase the number of undiagnosed true recent infected cases.

The positive predictive value (60.0\%) in our study for the TST test in comparison with the QTB (as the gold standard) was unacceptably low, but the negative predictive value (90.7\%) was acceptable. The $40.0 \%$ false positive results for TST might be due to BCG vaccination, remote infection or atypical Mycobacteria in older cases. Unnecessary treatment should be avoided by a complementary test such as the QTB to the TST $[15,16]$. Activated lymphocytes and effector memory cells producing interferon-gamma persist for a limited time in circulation once the antigen is cleared. So the QTB assay might reflect recent rather than remote $\mathrm{TB}$ infections. The $9.3 \%$ false negative rate for TST is important for early diagnosis $[15,16]$.
A blood test for M. tuberculosis has some theoretical advantages, even in developing countries. The QTB assay is a test without a booster effect for future tests and it produces results within 24 hours that do not require 2 patient visits. But the higher costs, greater practical inconvenience and the presence of indeterminate test results are limiting factors in the extensive use of QTB in large populations. Due to the reduced sensitivity of TST in subjects with recent M. tuberculosis exposure some authors suggest that for maximum sensitivity the 2 tests need to be used together, especially in BCG-vaccinated populations [6-8,15].

Some limitations of our study include the small numbers, especially in the lower age group $(<5$ years) and that subjects with negative results were not followed up for a longer duration (> 1 years) after the first visit.

\section{Conclusions}

The QTB assay, which can reflect recent rather than remote $\mathrm{TB}$ infections, is a better indicator for M. tuberculosis infection than TST in our country (with a BCG-vaccinated population). We suggest adding the interferon assay to skin test as a basis for the decision to perform chest X-ray or to start chemoprophylaxis treatment in the Islamic Republic of Iran, at least in younger individuals in close contact with cases. More extensive evaluation by the Ministry of Health is needed to change the screening and treatment programmes for young household contacts of active cases of pulmonary M. tuberculosis.

\section{Acknowledgements}

This study was supported by the Research Centre of Paediatric Infectious Diseases, Iran University of Medical Sciences. 


\section{References}

1. Walls T, Shingadia D. Global epidemiology of paediatric tuberculosis. Journal of Infection, 2004, 48:13-22.

2. Menzies D et al. Standardized treatment of active tuberculosis in patients with previous treatment and/or with mono-resistance to isoniazid: a systematic review and meta-analysis. PLoS Medicine, 2009, 6:e1000150.

3. Jasmer RM, Nahid P, Hopewell PC. Clinical practice. Latent tuberculosis infection. New England Journal of Medicine, 2002, 347:1860-1866.

4. Codecasa LR et al. Comparison of tuberculin skin test and Quantiferon immunological assay for latent tuberculosis infection. Monaldi Archives for Chest Disease, 2005, 63:158-162.

5. Rezai MS et al. Disseminated bacillus Calmette-Guerin infection after BCG vaccination. Journal of Tropical Pediatrics, 2008 54:413-416

6. Taggart EW et al. Evaluation of an in vitro assay for gamma interferon production in response to Mycobacterium tuberculosis infections. Clinical and Diagnostic Laboratory Immunology, 2004, 11:1089-1093.

7. Dogra $\mathrm{S}$ et al. Comparison of a whole blood interferon-gamma assay with tuberculin skin testing for the detection of tuberculosis infection in hospitalized children in rural India. Journal of Infection, 2007, 54:267-276.

8. Kariminia A et al. Comparison of QuantiFERON TB-G-test to TST for detecting latent tuberculosis infection in a high-incidence area containing BCG-vaccinated population. Journal of Evaluation in Clinical Practice, 2009, 15:148-151.

9. Taylor RE, Cant AJ, Clark JE. Potential effect of NICE tuberculosis guidelines on paediatric tuberculosis screening. Archives of Disease in Childhood, 2008, 93:200-203.
10. Friedman $\mathrm{LN}$ et al. High rate of negative results of tuberculin and QuantiFERON tests among individuals with a history of positive skin test results. Infection Control and Hospital Epidemiology, 2006, 27:436-441.

11. Mazurek GH et al. Detection of Mycobacterium tuberculosis infection in United States Navy recruits using the tuberculin skin test or whole-blood interferon-gamma release assays. Clinical Infectious Diseases, 2007, 45:826-836.

12. Marais BJ, Pai M. Recent advances in the diagnosis of childhood tuberculosis. Archives of Disease in Childhood, 2007, 92:446-452.

13. Ferrara $\mathrm{G}$ et al. Use in routine clinical practice of two commercial blood tests for diagnosis of infection with Мyсоbacterium tuberculosis: a prospective study. Lancet, 2006, 367:1328-1334.

14. Hill PC et al. Comparison of enzyme-linked immunospot assay and tuberculin skin test in healthy children exposed to $\mathrm{Myco-}$ bacterium tuberculosis. Pediatrics, 2006, 117:1542-1548.

15. Diel $\mathrm{R}$ et al. Tuberculosis contact investigation with a new, specific blood test in a low-incidence population containing a high proportion of BCG-vaccinated persons. Respiratory Research, 2006, 7:77.

16. Diel $\mathrm{R}$ et al. Predictive value of a whole blood IFN-assay for the development of active tuberculosis disease after recent infection with Mycobacterium tuberculosis. American Journal of Research and Critical Care Medicine, 2008, 177:1164-1170. 